\title{
Search for high-redshift blazars with Fermi/LAT
}

\author{
Michael Kreter ${ }^{\star a}$, Matthias Kadler ${ }^{b}$, Felicia Krauß ${ }^{c}$, Sara Buson ${ }^{b}$, Roopesh Ojha ${ }^{d}$, for \\ the Fermi/LAT Collaboration, Jörn Wilms ${ }^{e}$ and Markus Böttcher ${ }^{a}$ \\ ${ }^{a}$ Centre for Space Research, North-West University, Private Bag X6001, Potchefstroom 2520, \\ South Africa \\ ${ }^{b}$ Lehrstuhl für Astronomie, Universität Würzburg, Emil-Fischer-Straße 31, 97074 Würzburg, \\ Germany \\ ${ }^{c}$ GRAPPA \& Anton Pannekoek Institute for Astronomy, University of Amsterdam, Science \\ Park 904, 1098 XH Amsterdam, The Netherlands \\ ${ }^{d}$ NASA Goddard Space Flight Center, Greenbelt, MD 20771, USA \\ ${ }^{e}$ Dr. Remeis Sternwarte \& ECAP, Universität Erlangen-Nürnberg, Sternwartstraße 7, 96049 \\ Bamberg, Germany \\ michaelekreter.org
}

High-redshift blazars $(z \geq 2.5)$ are one of the most powerful classes of $\gamma$-ray sources in the Universe. These objects possess the highest jet powers and luminosities and have black-hole masses often in excess of $10^{9}$ solar masses. In addition, high-redshift blazars are important cosmological probes and serve as test objects for blazar evolution models. Due to their large distance, their high-energy emission peaks are often downshifted to energies below the GeV range, which makes them difficult to study with Fermi/LAT and only the very brightest objects are detectable. Hence, only a small number of high-redshift blazars could be detected with Fermi/LAT so far. In this work, we present a strategy to increase the detection statistics at redshift $z \geq 2.5$ via a search for flaring events in high-redshift $\gamma$-ray blazars whose long-term flux is just below the sensitivity limit of Fermi/LAT. Seven previously GeV undetected high-redshift blazars have been identified from their bright monthly outburst periods, while more detections are expected in the future.

36th International Cosmic Ray Conference -ICRC2019-

July 24th - August 1st, 2019

Madison, WI, U.S.A.

${ }^{*}$ Speaker. 


\section{Introduction}

Blazars, as a subclass of active galactic nuclei (AGN) are characterized by jets pointing towards the observer. These sources are among the most powerful objects in the Universe. Their broadband emission is dominated by relativistic jets, especially at $\gamma$-rays where the high-energy component of the spectral energy distribution (SED) peaks in the $\mathrm{MeV}$ to $\mathrm{GeV}$ regime. The Large Area Telescope (LAT) onboard the Fermi satellite has detected thousands of blazars, confirming that they dominate the $\gamma$-ray sky [1]. However, high-redshift $(z \geq 2.5)$ blazars are outnumbered in Fermi catalogs by their nearby counterparts. $[1,2]$. This is due to a shift of the high-energy emission towards energies below the LAT band, both because of cosmological redshift and following the blazar sequence [3]. In addition, absorption in the EBL leads to an efficient degrading of highenergy $\gamma$-rays below the detection threshold of Fermi/LAT [4, 5]. Although commonly represented in the radio and X-ray regime [6,7], these blazars are rare $\gamma$-ray emitters, accounting for less than $10 \%$ of the total AGN population observed by Fermi/LAT. High-redshift blazars are of particular interest in the field of $\gamma$-ray Astronomy, as they are the most powerful (non-transient) astrophysical sources ever detected by Fermi/LAT. These sources possess the highest jet powers and accretion luminosities and have black-hole masses often in excess of $10^{9} M_{\odot}$ [8], [9]. High-redshift blazars are important as cosmological probes and serve as test objects for blazar evolution models [10, 11, 12]. This directly implies, that the detection of unknown high-z blazars would constrain models of blazar evolution, as the extreme distance would limit the available time for these objects to grow to such enormous black hole masses. In fact, only a small number of high-redshift blazars has been detected by Fermi/LAT, some of them only because of bright flaring states [13]. Recently, five new high-redshift blazars have been identified by Fermi/LAT [4], making NVSS J151002+570243 the most distant known $\gamma$-ray emitting blazar at $z=4.31$. These detections were only possible due to the upgrade of Fermi/LAT to the Pass 8 analysis and a systematic analysis of a large blazar sample. In this work, we introduce a new method to search for hitherto undetected high-redshift $\gamma$-ray emitting blazars, which are too faint to be detected significantly (test statistic (TS) $\geq 25$ ) on long-term periods typically considered for catalogs, but can show up as significant sources during shorter $(\sim$ monthly) periods of increased activity.

\section{Detection Strategy and Sample Selection}

Flaring states from previously $\gamma$-ray unknown high-redshift blazars have been detected several times by Fermi/LAT $[14,15,16]$. Such periods of enhanced activity typically only last for several days, leading to a significant detection (TS $\geq 25$ ) due to a drastic background reduction, compared to long-term averaged fluxes used in catalogs. Figure 1 shows a ten-year Fermi/LAT, $\gamma$-ray light curve of the high-redshift $(\mathrm{z}=2.83)$ blazar PKS 0438-43 in the energy range of $100 \mathrm{MeV}$ to $300 \mathrm{GeV}$. This source was undetected in the third Fermi/LAT Point Source catalog and is detected at $\mathrm{GeV}$ energies only due to its bright flare in $2016^{1}$ [14].

In this work, we perform a systematic search for $\gamma$-ray undetected high-redshift blazars, by targeting a sample of 176 blazars with a redshift of $z \geq 2.5$ and a radio flux density of more than

\footnotetext{
${ }^{1}$ PKS 0438-43 is now included in the Fourth Fermi/LAT Point Source catalog (4FGL)
} 


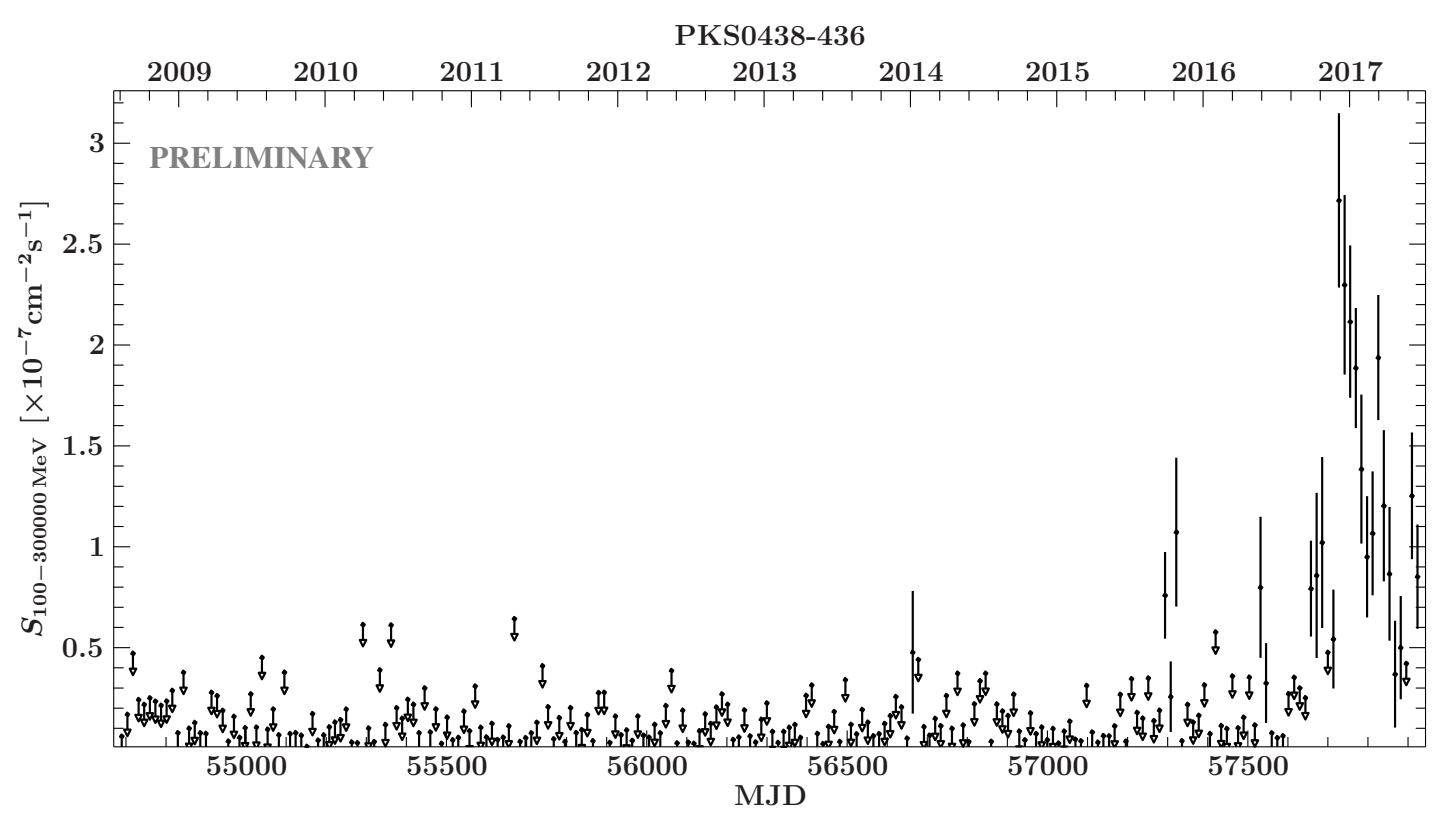

Figure 1: Fermi/LAT $\gamma$-ray light curve of PKS 0438-43 over $\sim$ ten-years of continuous observations. The light curve is shown for the energy range of $100 \mathrm{MeV}$ to $300 \mathrm{GeV}$. This source has been detected in December 2016 during a bright flare.

$50 \mathrm{mJy}$, taken from the Roma BZCAT Multifrequency Catalogue of Blazars ${ }^{2}$ and the SHAO list of high-redshift radio-loud quasars ${ }^{3}$. These sources are selected because of the expected correlation between radio and $\gamma$-ray flux $[17,18]$. The total sample consists of 169 BZCAT blazars, as well as seven SHAO sources. Monthly binned Fermi/LAT $\gamma$-ray light curves in the time range from 2008 August 4 to 2019 April 4 and energy range of $100 \mathrm{MeV}$ to $300 \mathrm{GeV}$ are calculated, while the time binning of 30 days has been chosen to identify periods which would not have been followed up by the Fermi/LAT Flare Advocate service and keep the computing effort at a reasonable level.

\subsection{Detection Potential}

In order to estimate the number of high-redshift blazars potentially detectable by this approach, a $\log N-\log S_{\max }$ diagram of known high-redshift $\gamma$-ray blazars is shown in Fig. 2. We calculate the cumulative number of sources per square degree with a redshift $z \geq 2.5$, taken from the third catalog of active galactic nuclei detected by Fermi/LAT (3LAC; [19]). A power-law fit is used for parametrization, allowing to extrapolate the number of expected high-redshift blazars towards lower fluxes in the energy range $1 \mathrm{GeV}-100 \mathrm{GeV}^{4}$. For PKS 0438-43, which was not detected in the 3LAC, we calculate the corresponding upper limit to be $4 \times 10^{-11} \frac{\mathrm{ph}}{\mathrm{cm}^{2} \mathrm{~s}}$. At this photon flux limit, approximately 140 high-redshift blazars should be detectable by our approach, of which only 19 are known in the 3LAC. In order to estimate the amount of detectable high-redshift blazars showing periods of enhanced activity on monthly time scales, it is important to understand the expected false-positive detection rate for this kind of analysis.

\footnotetext{
${ }^{2}$ http://www.asdc.asi.it/bzcat/

${ }^{3}$ http://202.127.29.4/CRATIV/en/high_z.html

${ }^{4}$ These energy range has been used in the $3 \mathrm{LAC}$
} 


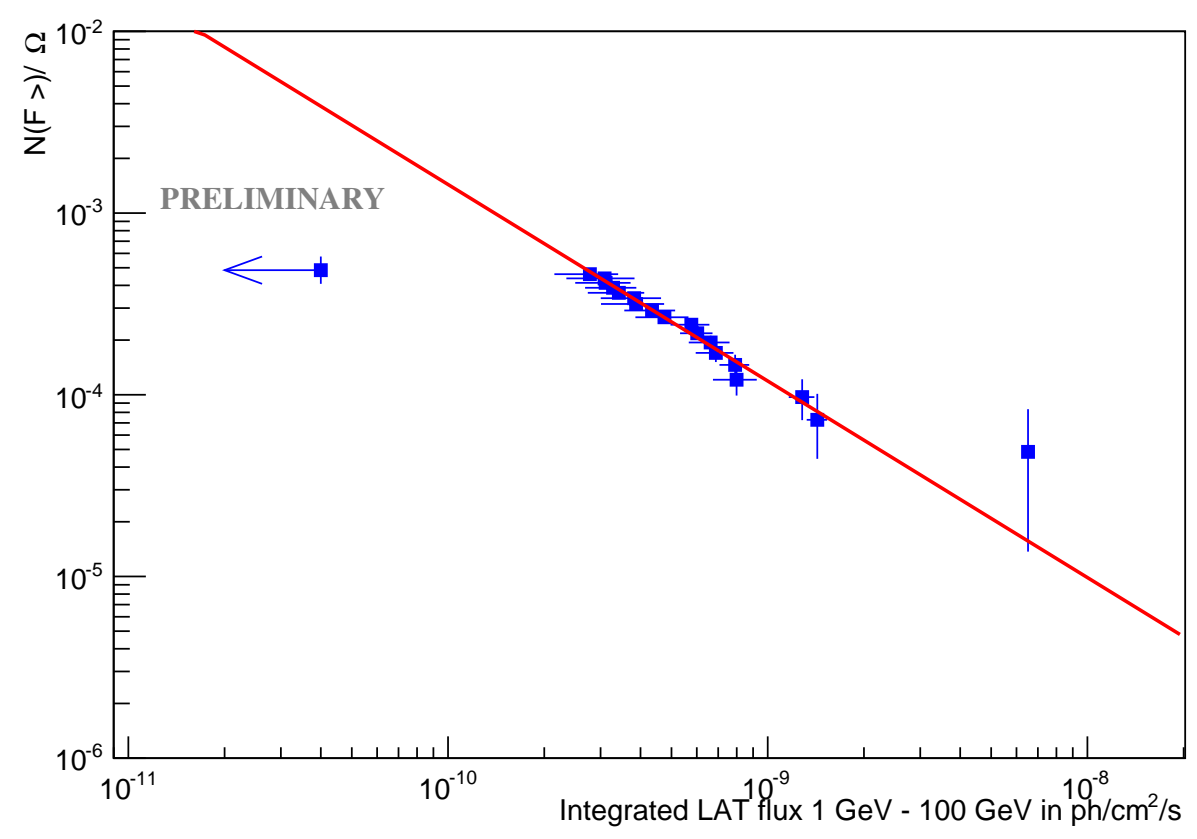

Figure 2: $\log N-\log S_{\max }$ digram of blazars taken from the third catalog of active galactic nuclei detected by Fermi/LAT (3LAC). The plot shows the cumulative number of sources normalized per square degree with a redshift $\mathrm{z} \geq 2.5$, using statistical uncertainties only. The power-law fit $(N=(1.92 \pm 0.68) \times$ $\left.10^{-14} \times S^{-1.079 \pm 0.097}\right)$ is used for parametrization. The arrow bar refers to the corresponding upper limit from PKS 0438-43.

\subsection{Background Determination}

For monthly intervals detected at a test statistic level of 25 or more, the chance for a false positive detection is about $5 \times 10^{-7} \%$. Thus, $\gamma$-ray detections at this significance level are considered as trustworthy. Multiple ( $\mathrm{TS}<25$ ) monthly detections from an unknown emitter, however, suggest the presence of a new source, even if non of the monthly intervals have been detected above the required (TS $>25$ ) threshold. In order to account for these sources, we preform a simi$1 a r^{5}$ Fermi/LAT light curve analysis on a large sample of blank-sky positions. These positions have been randomly selected and require the next known 4FGL source to be at least $2^{\circ}$ apart from the studied sky position. Figure. 3 shows the test statistic distribution of monthly intervals for a total of 50 studied blank-sky positions, while the TS distribution of 100 known blazars ${ }^{6}$ is shown in Fig 4. By comparing these two distributions for each test statistic bin, we derive the Poisson probability for a false positive detection to be:

$$
\int P\left(N_{s} \mid \mu_{b}\right) d N_{s}
$$

\footnotetext{
${ }^{5}$ The same energy and time range and binning has been used.

${ }^{6}$ undetected by Fermi/LAT
} 


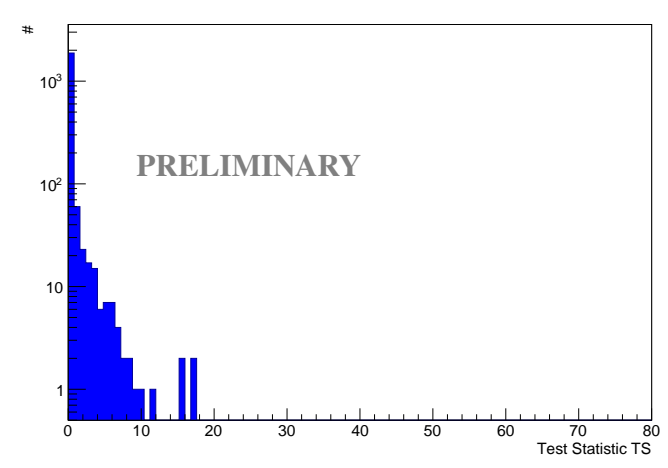

Figure 3: Test statistic distribution of a sample of 50 blank-sky positions. This histogram represents the background fluctuation for the considered Fermi/LAT variability analysis.

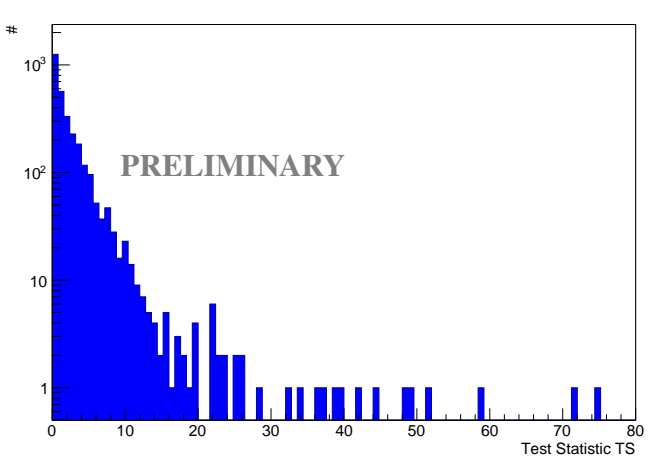

Figure 4: Test statistic distribution of $100 \gamma$-ray undetected blazar positions selected according to Sect.2. 21 monthly intervals are identified with a significance of $\mathrm{TS} \geq 25$, while at a TS $\geq 9$ level 119 intervals are detected.

$N_{s}$ corresponds to the number of "signal" monthly intervals detected from the directions of known blazars, while $\mu_{b}$ corresponds to the amount of random fluctuations ${ }^{7}$ calculated from a large sample of blank-sky positions. The total probability of multiple monthly intervals to be of purely statistical nature thus is given by:

$$
\prod_{i} P_{i}\left(N_{i b} \mid \mu_{i b}\right)
$$

The product is defined over all bins in Fig. 4, which show at least one entry with a TS $\geq 9$.

\section{Detected (new) $\gamma$-ray sources}

\subsection{TS $>25$ detections}

A total of 21 monthly intervals from seven different blazars have been identified with a test statistic of TS $\geq 25$. Table 1 lists the relevant parameters of these sources. Blazars marked by a star indicate sources, first identified in the recently published 4FGL catalog [1]. Three additional new $\gamma$-ray undetected high-z blazars have been identified, making 5BZQ J0246+1823 with a redshift of $z=3.59$ the fourth most distant blazar known to Fermi/LAT [4].

\subsection{TS $\geq 9$ detections}

At a test statistic level of TS $\geq 9$ a total of 119 monthly intervals have been identified from a sample of 57 high-z blazars. Excluding blazars which have already been detected significantly (TS $>25)$, this results in a sample of 98 monthly intervals detected from 50 blazars. Based on the 50 studied blank-sky positions ${ }^{8}$, only five monthly intervals showed a fluctuation above the TS $>9$ threshold. By considering the total amount of studied blank-sky intervals, only a fraction of

$$
5 / 6000 \approx 8.3 \times 10^{-4} \approx 3.3 \sigma
$$

\footnotetext{
${ }^{7}$ at the selected or larger test statistic

8 which include a total of 120 bins x 50 positions $=6000$ studied monthly intervals
} 
Table 1: Identified high-redshift blazars which are at least detected in a single monthly interval with a $\mathrm{TS} \geq 25$. Sources listed in the 4FGL are marked by a star.

\begin{tabular}{ccccc}
$\begin{array}{c}\text { Source } \\
\text { Name }\end{array}$ & $\begin{array}{c}\text { RA } \\
\text { J2000 }\end{array}$ & $\begin{array}{c}\text { DEC } \\
\text { J2000 }\end{array}$ & $\mathbf{z}$ & $\begin{array}{c}\text { Detections } \\
\text { TS } \geq \mathbf{2 5}\end{array}$ \\
\hline 5BZQ J0009+0625 & 2.32 & 6.43 & 2.69 & 1 \\
5BZQ J0225+1846 $^{\star}$ & 36.27 & 18.78 & 2.69 & 2 \\
5BZQ J0339-0133 & 54.75 & -1.55 & 3.20 & 5 \\
5BZQ J0246+1823 & 41.54 & 18.39 & 3.59 & 1 \\
5BZQ J0434-4355 & 68.51 & -43.93 & 2.65 & 2 \\
5BZQ J0733+0456 & 113.49 & 4.94 & 3.01 & 7 \\
5BZQ J1127+5650 & 171.92 & 56.84 & 2.89 & 3 \\
& & & & \\
\hline
\end{tabular}

monthly intervals showed a statistical fluctuation with a TS $\geq 9$ level. Thus, we can conclude that from our sample of 100 studied blazars, 50 sources indeed emitted $\gamma$-rays at a significance of at least $3.3 \sigma$.

\section{Discussion and Outlook}

In this work, we identified three new high-redshift blazars, which were too faint to be detected on long-term time scales of several years, but showed significant $\gamma$-ray activity at a TS $\geq 25$ level on monthly time scales. Four additional ${ }^{9}$ blazars had been unknown to the third Fermi/LAT Point Source Catalog (3FGL), but showed flaring episodes in the recent years and are therefore included as new sources in the 4FGL. While still being closer than the most distant blazar identified in [4], the blazar 5BZQ J0246 +1823 at a redshift of $z=3.59$ represents the farthest, significantly detected new $\gamma$-ray blazar identified in this work..

By comparing the observed test statistic of each $\gamma$-ray undetected blazar position to the background fluctuation in Fig. 3, we showed that a TS $\geq 9$ detection corresponds to a significance level of $\sim 3 \sigma$. As half of our studied sample has been detected in at least one monthly interval with a $\mathrm{TS} \geq 9$, this implies that the statistic of $\gamma$-ray detected high-z blazars can be significantly increased by searching for periods of enhanced activity. Especially the blazar 5BZQ J1026+2542, at a redshift of $z=5.28$ is a promising candidate for one of the most distant $\gamma$-ray blazars ${ }^{10}$. More potential high-redshift blazar detections are expected from the full sample, together with a more precise background estimate by increasing the number of blank-sky positions by a factor between two and four. In addition, the detection potential of high-redshift blazars can be increased by evaluating a possible $\gamma$-ray detection for all $z \geq 2.5$ blazars in the Roma BZCAT Multifrequency Catalogue and the SHAO list of high-redshift loud quasars, instead of just focusing on the radio brightest sources. Nevertheless, the new identified $\gamma$-ray blazars will need to be studied in detail. A dedicated analysis will be performed in the future in order to study their spectral properties in the $\mathrm{MeV}$ to $\mathrm{GeV}$ regime.

\footnotetext{
${ }^{9}$ marked by star

${ }^{10}$ This blazar is currently detected at a $\mathrm{TS} \approx 12$ level.
} 


\section{References}

[1] The Fermi-LAT collaboration, Fermi Large Area Telescope Fourth Source Catalog, arXiv e-prints (2019) [arXiv:1902.10045]

[2] Acero et. al., Fermi Large Area Telescope Third Source Catalog, ApJS 218 (2015) 23 [arXiv:1501.02003]

[3] Fossati et. al., A unifying view of the spectral energy distributions of blazars, MNRAS 299 (1998) 433-448 [arXiv:astro-ph/9804103]

[4] Ackermann et. al., Gamma-Ray Blazars within the First 2 Billion Years, ApJ 837 (2017) L5 [arXiv:1702.04006]

[5] H.E.S.S. Collaboration et. al., Measurement of the extragalactic background light imprint on the spectra of the brightest blazars observed with H.E.S.S., A\&A 550 (2013) A4 [arXiv:1212.3409]

[6] Condon et. al., The NRAO VLA Sky Survey, AJ 115 (1998) 1693-1716

[7] Nasa and Heasarc, VizieR Online Data Catalog: Swift Master Catalog (HEASARC, 2004-), VizieR Online Data Catalog 1 (2018)

[8] Bloemen et. al., New COMPTEL gamma-ray source (GRO J0516-609) near PKS 0506-612/0522-611: first evidence for "MeV Blazars, A\&A 293 (1995)

[9] Ghisellini et. al., Chasing the heaviest black holes of jetted active galactic nuclei, MNRAS 405 (2010) 387-400 [arXiv:0912.0001]

[10] M. Ajello, Cosmological evolution of blazars: new findings from the Swift/BAT and Fermi/LAT surveys, TeV Particle Astrophysics 2009 (2009) 81

[11] Ajello et. al., The Luminosity Function of Fermi-detected Flat-spectrum Radio Quasars, ApJ 751 (2012) 108 [arXiv:1110.3787]

[12] Ajello et. al., The Cosmic Evolution of Fermi BL Lacertae Objects, ApJ 780 (2014) 73 [arXiv:1310.0006]

[13] Paliya et. al., Detection of a Gamma-Ray Flare from the High-redshift Blazar DA 193, ApJ 871 (2019) 211 [arXiv:1812.07350]

[14] C. Cheung, Fermi LAT Detection of a New Gamma-ray Source Associated with High-Redshift Blazar PKS 0438-43, The Astronomer's Telegram 9854 (2016)

[15] C. Cheung, Fermi LAT detection of a GeV flare from High-redshift Blazar PKS 0537-286, The Astronomer's Telegram 10356 (2017)

[16] R. Angioni, Fermi-LAT detection of a new gamma-ray source associated with the high-redshift FSRQ TXS 0552+398, The Astronomer's Telegram 11137 (2018)

[17] Ghirlanda et. al., Correlation of Fermi Large Area Telescope sources with the 20-GHz Australia Telescope Compact Array radio survey, MNRAS 407 (2010) 791-803 [arXiv:1003.5163]

[18] Ackermann et. al., The Radio/Gamma-Ray Connection in Active Galactic Nuclei in the Era of the Fermi Large Area Telescope, ApJ 741 (2011) 30 [arXiv:1108.0501]

[19] Ackermann et. al., The Third Catalog of Active Galactic Nuclei Detected by the Fermi Large Area Telescope, ApJ 810 (2011) 14 [arXiv:1501.06054]

[20] M. Kreter, Targeting the mystery of extragalactic neutrino sources - A Multi-Messenger Window to the Extreme Universe -, PhD-thesis Würzburg, 2019 